\title{
SiMEA : a Framework for simulating neurons on Multi-Electrode Array
}

\section{Andalibi, Vafa}

IEEE

2016

Andalibi , V , Aaltonen , T , Christophe , F \& Mikkonen , T 2016 , SiMEA : a Framework for simulating neurons on Multi-Electrode Array . in J Patton, R Barbieri , J Ji , E Jabbari , S Dokos, R Mukkamala, D Guiraud, E Jovanov, Y Dhaher, D Panescu, M Vangils, B Wheeler \& AP Dhawan (eds), IEEE Engineering in Medicine and Biology Society Conference Proceedings : 2016 38TH ANNUAL INTERNATIONAL CONFERENCE OF THE IEEE ENGINEERING IN MEDICINE AND BIOLOGY SOCIETY (EMBC) . vol. 38 , 7592087 , IEEE Engineering in Medicine and Biology Society Conference Proceedings, IEEE, pp. 5965-5968 , Annual International Conference of the IEEE Engineering in Medicine and Biology Society , Lake Buena Vista , United States , 16/08/2016 . https://doi.org/10.1109/EMBC.2016.7592087

http://hdl.handle.net/10138/189271

https://doi.org/10.1109/EMBC.2016.7592087

unspecified

publishedVersion

Downloaded from Helda, University of Helsinki institutional repository.

This is an electronic reprint of the original article.

This reprint may differ from the original in pagination and typographic detail.

Please cite the original version. 


\title{
SiMEA: a Framework for simulating neurons on Multi-Electrode Array*
}

\author{
Vafa Andalibi, Student Member, IEEE, Timo Aaltonen, Francois Christophe, and Tommi Mikkonen
}

\begin{abstract}
A Multi-Electrode Array (MEA) is a practical device for recording the extracellular activity of in-vitro biological culture. Such culture - for instance neurons - is prone to mistakes leading to irrelevant recordings or no recording at all. Additionally, with the expenses generated by in-vitro culture, minimizing risks is a must. This paper proposes a framework designed and implemented for simulating the spatial positioning of neuronal cultures on a MEA. The framework serves as a sandbox for researchers to simulate the model of their MEA experiments before its eventual in-vitro implementation. The framework enables simulating the density of the plated culture, the death of cells over time, choosing diverse reconstructed morphologies of cells, and simulating their spiking activity in interaction with Brian2 simulator.
\end{abstract}

\section{INTRODUCTION}

The development of Multi-Electrode Arrays (MEA) for recording the extracellular activity of in-vitro cultures has taken an important role in understanding the biological organization of neuronal cells into networks [1], connectivity changes within this network [2], and its functional behavior [3], [4].

MEA systems can be used as a tool for recording the extracellular electrophysiological activity in in-vitro environment, which assists researchers in several areas, like studying brain function [5], and investigating the retinal circuitry to visual cortex [6]. In engineering domains, MEA systems are is used as an interface between biological parts and technical systems, resulting in bio-integrated systems [7]. Such interfacing is realized by stimulating the neurons and recording their extracellular activity in response to stimulation. Examples of recent successes in this interfacing by utilizing MEA are Lego Mindstorm robot [8], and hybrid neurorobot system [9].

Unfortunately, the use and maintenance of both cultured and dissociated neurons in a MEA are laborious tasks. Moreover, several risk factors, such as susceptibility to infection, long maturation duration, and hyperosmolality

\footnotetext{
*This work is supported by the Academy of Finland under the project "Bio-integrated Software Development for Adaptive Sensor Networks", Project number 278882.

V. Andalibi is with Clinical neurosciences, Neurology, University of Helsinki and Helsinki University Hospital, Helsinki, Finland (e-mail: vafa.andalibi@helsinki.fi).

T. Aaltonen is with Department of Pervasive Computing, Tampere University of Technology, Tampere, Finland (e-mail: timo.aaltonen@tut.fi).

F. Christophe is with Department of Pervasive Computing, Tampere University of Technology, Tampere, Finland (corresponding author to provide e-mail: francois.christophe(tut.fi).

T. Mikkonen Department of Pervasive Computing, Tampere University of Technology, Tampere, Finland (e-mail: tommi.mikkonen(atut.fi).
}

resulting from evaporation in the medium, are specifically associated with cultured neuronal networks. Only cultures that avoid these issues are used in recording and stimulation but only for a short period of their lifetime. Despite the efforts to keep neural cultures viable for long-terms [10], or to record and stimulate while keeping cultures in the incubator[11], the average lifetime of in-vitro neuronal cultures is slightly longer than a month.

To address the above issue, we present the design and implementation of a simulation framework called SiMEA for representing the physical and electrical behavior of neuronal cultures on a MEA. There are several differences between the approach taken in SiMEA and that of older studies, e.g. Cultured Neuron Simulator (CNS) [12]: the main difference is that neural connections in the latter are modeled statistically whereas in SiMEA they are determined based on neuronal morphologies. Other studies focus either on simulation of MEA hardware [13], [14] or on signaling [15], [16].

This paper is organized as follows: Section II outlines the main components of the SiMEA as well as the interaction between them. In Section III, intermediate results of the core components as well as the final output of SiMEA are illustrated. Finally, Section IV presents our conclusion.

\section{METHOD}

SiMEA consists of five main components: cell plating, cell death, morphology, interpreter, and brian2 simulator [17] containing neuron model, and neuroplasticity models. The relations between these components are illustrated in Figure 1. In following subsections, each of the SiMEA components are described in more details.

\section{A. Cell Plating}

For the purpose of simulating the density of cells plated on a MEA, Shultz proposed the use of midpoint displacement fractal algorithm in his development of named Cultured Neuron Simulator (CNS) [12]. Using this algorithm, however, the cells would mostly occupy locations near the edges of the MEA plate. In this study, we adopted a different approach, entitled two-level inversed diamond-square. This algorithm is based on the inversion of diamond-square algorithm [18], the successive of midpoint displacement fractal algorithm that also suffers from the same issue as the midpoint displacement algorithm. In this approach, the inversed diamond-square algorithm is first applied by considering the soma area 10 times larger than its value in the simulator, resulting in a smoother distribution over a wider area. Based on this uniform distribution, the exact position of the soma within its wider box is determined by the application of the diamond square within this box. For this 


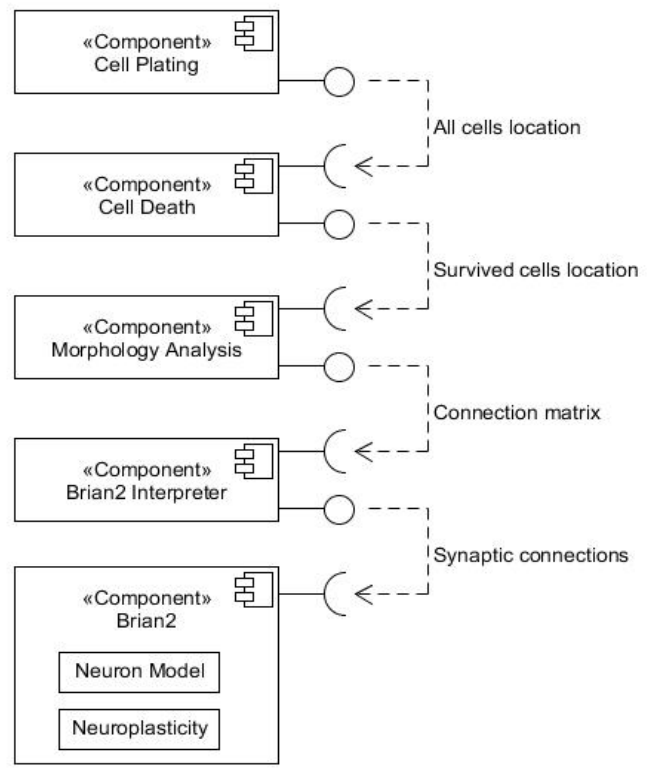

Figure 1. internal components of SiMEA

reason, this algorithm is named "two-level" inversed diamond-square.

Figure 2 presents the results from the three algorithms: b) diamond square, c) inversed diamond-square, and d) twolevel inversed diamond-square. These results are compared with a grayscale version of fluorescence imaging of neuronal culture in a real MEA plate shown in Fig.2 a). This plating comparison is realized with the image processing software Image $^{1}$ in the following way. Each figure is divided into a $10 \times 10$ grid and the number of neurons in each grid cell is calculated and corresponding histogram is drawn. Next, the histogram of each algorithm is compared with the fluorescence image using the Kullback-Leibler divergence measure and the similarity of the result of the algorithms with a real MEA plate are determined.

\section{B. Cell Death}

During the first 17 Days in Vitro (DIV) and before final formation of the network, $45-60 \%$ of the cells will die [19]. This phenomenon has an important effect on development of the culture and should be taken into account. However, the runtime for 17 days of simulation time is enormous. Hence, by default the starting point of the simulation will be the 17 th DIV, or when indices of the dead cells are all determined. These indices are determined based on a 2-D random sampling over time and indices of neurons remaining alive.

\section{Morphology Analysis}

Without consideration of the form and structure of neurons, i.e. their morphology, neurons would be represented solely as square or circular somas. In this case, the strength of connections between neurons would be determined as a function of their distance. Taking neuromorphological aspects into account offers the possibility to compute neuronal connections based on the position of axons and dendrites. Furthermore, the connectivity between neurons is determined based on the intersection of one cell's axonal

\footnotetext{
${ }^{1}$ http://imagej.nih.gov/ij/
}

branches with dendrites of another cell. Morphologies of neurons are downloaded from NeuroMorpho ${ }^{2}$ database.

\section{Brian2 Interpreter}

The role of this component in the framework is to convert morphological and structural data about the plated neuronal network into useable data for simulating the physiological behavior of this network. This component works as an interface between the SiMEA framework and the Brian2 simulator. For each neuron in the plated network, converted data expresses the following data: the behavioral type of this neuron, i.e. excitatory or inhibitory, the identity of neurons it is connected to in the network, and the number of synapses between them.

Once converted, this data is used to simulate the dynamic behavior of the plated network in Brian2 as shown in subsection E.

\section{E. Neuron and Neuroplasticity Model in Brian2}

The physiological behavior of a single neuron in this framework is modeled with Izhikevich simple model of neuronal behavior [9]. In this study, excitatory and inhibitory neurons are modeled respectively with the parameters of Regular Spiking and Fast Spiking neurons as expressed in [20].

The initial connection strength between two neurons is modeled based on the number of synapses found from the morphological analysis component. Synaptic changes are modeled depending on the type of the presynaptic neuron with AMPA- and GABA-ergic synapses for excitatory and inhibitory neurons, respectively. In addition, Spike Timing Dependent Plasticity (STDP) is modeled between neurons as expressed in [21], [22] with equations 1 and 2.

$$
\Delta w_{j}=\sum_{f=1}^{N} \sum_{n=1}^{N} W\left(t^{n}-t_{j}^{f}\right)
$$

In Equation $1, \Delta w_{j}$ defines the strength change of synapse $j$ based on the summation of STDP function $W\left(t^{n}-t_{j}^{f}\right)$. In this function, $t^{n}$ and $t_{j}^{f}$ represent the time of nth spike of the postsynaptic neuron and the time of $f$ th spike of the presynaptic neuron. The function $\mathrm{W}(\mathrm{x})$ is usually defined as Equation .

$$
W(x)= \begin{cases}A_{+} e^{-\frac{x}{\tau_{+}}}, & x>0 \\ A_{-} e^{-\frac{x}{\tau_{-}}}, & x \leq 0\end{cases}
$$

where $A_{+}, A_{-}, \tau_{+}$and $\tau_{-}$determine the magnitude and dropoff of each side of the exponentials.

\section{RESULTS}

Starting with plating and cell distribution, Figure 2 shows the cell distribution resulting from diamond-square algorithm, inversed diamond square algorithm, two level inversed diamond square algorithm and a grayscale version of fluorescence image of neuronal culture in a real MEA plate. These results from algorithms are compared to real distribution of cells on a MEA plate with the KullbackLeibler divergence measure applied on the four

\footnotetext{
${ }^{2}$ http://neuromorpho.org/
} 

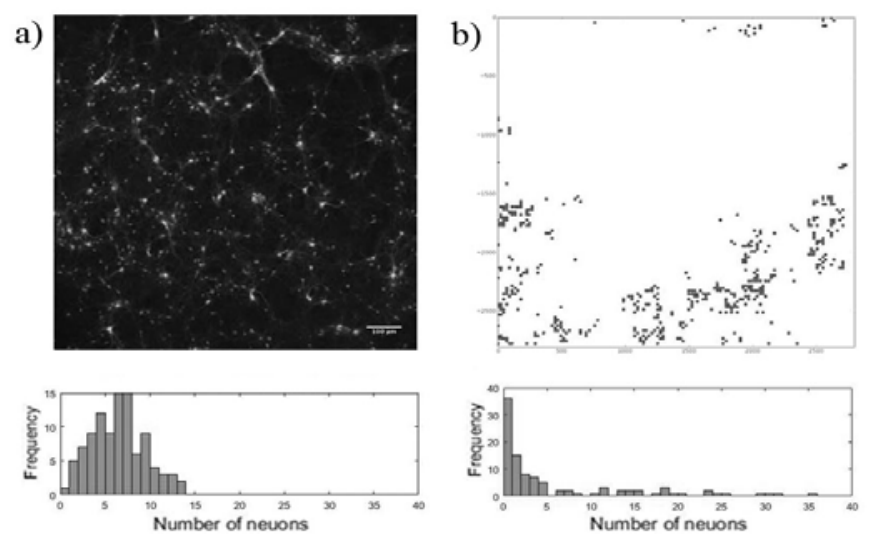

c)
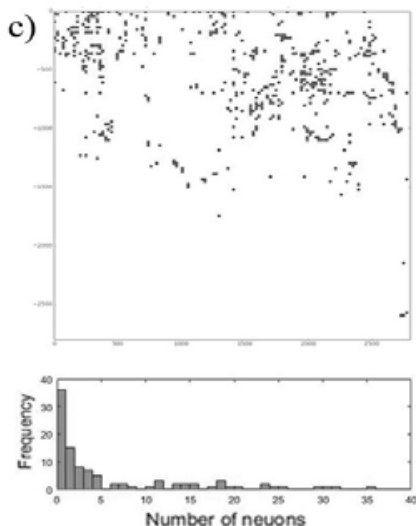

d)
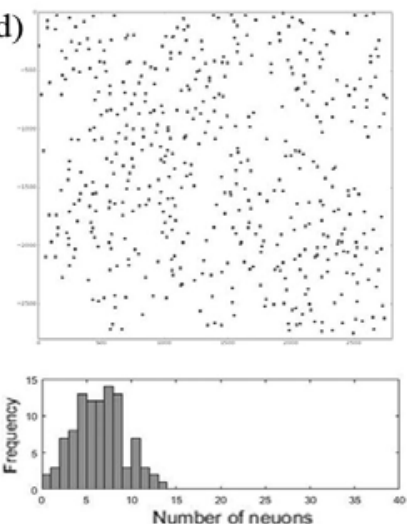

Figure 2. Comparison between real plating on a MEA and simulated plating a) Gray-scale fluorescence image of neurons in a MEA from [23]. Plating simulated with b) diamond-square algorithm, c) inversed diamond square algorithm d) two-level inversed diamond square algorithm

corresponding distribution histograms shown in Fig. 2. The results from the Kullback-Leibler divergence measure presented in Table I confirm a significant similarity between the two level inversed diamond square algorithm and the real cell distribution (distance of 0.0893 between them).

After determining the location of the survived cells in the MEA plate, the morphology of the neurons is generated based on their cell type, soma position and rotation angle. The intersect finder algorithm is then applied on neurons in a pairwise manner, generating the connection map. Figure 3 shows the morphology and connections points of a very small network with 10 neurons.

By inputting the information about the neuron groups, e.g. number of neuron groups and type of each neuron group, as well as connection map data into the Brian2 using the brian2 interpreter component, a dynamic simulation of the network activity is computed. Figure 4 shows the output of the framework for a MEA initialized with 1000 neurons, 493 of which survived after applying the cell death. This network consists of $70 \%$ excitatory pyramidal cells of five references in NeuroMorpho, i.e. C031097B-P3, C300898C-P2, I03481, D20D and D27B, and 30\% inhibitory basket cells of five references, i.e. BE49B, BE23B, C010600A2, C010600C1,

TABLE I. KULLBACK-LEIBLER DIVERGENCE MEASURES RESULTED FROM COMPARING THE ALGORITHM RESULTS WITH THE REAL MEA. GREATER VALUE INDICATES HIGHER DIVERGENCE, HENCE LESS SIMILARITY.

\begin{tabular}{|l|c|c|c|}
\hline Kullback- & \multicolumn{3}{|c|}{ Algorithm } \\
\cline { 2 - 4 } $\begin{array}{c}\text { Leibler } \\
\text { divergence }\end{array}$ & $\begin{array}{c}\text { Diamond } \\
\text { Square }\end{array}$ & $\begin{array}{c}\text { Inversed Diamond } \\
\text { Square }\end{array}$ & $\begin{array}{c}\text { Two-level inversed } \\
\text { diamond-square }\end{array}$ \\
\hline Measure & 1.6960 & 1.2918 & 0.0893 \\
\hline
\end{tabular}

and C070600A4. The framework allows an easy addition of more morphologies expressed in SWC format as well as their associated firing patterns (e.g. bursting).

\section{CONCLUSION}

This paper presented the design and implementation of a framework allowing multiple simulation viewpoints on a model of in-vitro neuronal networks cultured on MEA. These viewpoints enable a realistic visualization of the spatial distribution of cells within a MEA; the distribution of cells with diverse morphologies; the creation of a neuronal network composed of neuronal cells of various morphologies and their associated firing patterns; and the simulation of the dynamical activity of this random recurrent network.

Our results show relevant similarities to in-vitro experiments on MEA in three main areas. First, the density distribution of neurons in the plate simulated by the proposed two-level inversed diamond square algorithm achieves a high similarity with real in-vitro plating (distance between both distributions of 0.0893, based on Kullback_Leibler divergence measure). Second, neuron shapes and connection formation in the model are based on the reconstruction of real morphologies. Third, the dynamic activity of the network simulated with SiMEA shows intrinsic synchronous activity close to MEA.

The framework is easily configurable for the simulation of experiments with other in-vitro cultures than the neuronal culture presented in this paper. The source code is freely available on github ${ }^{4}$. Our future plans for this framework

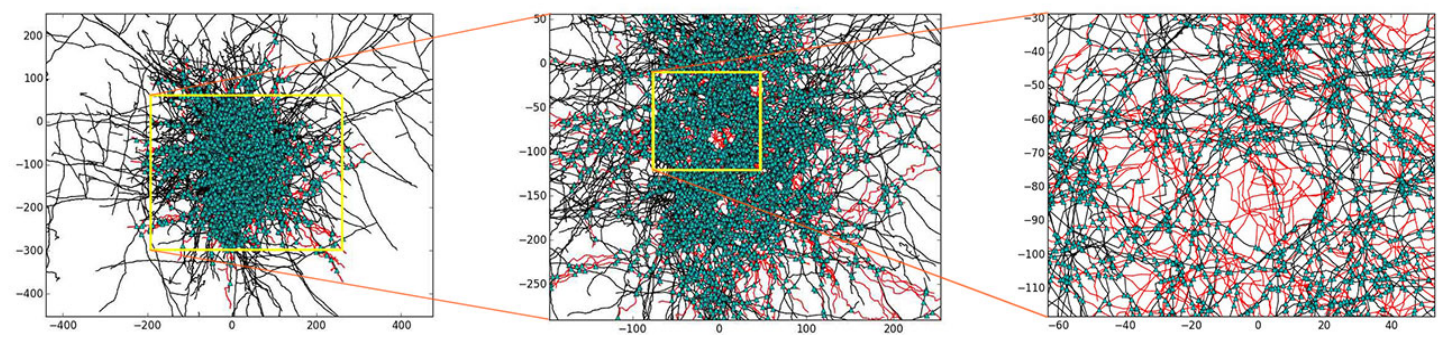

Figure 3. Visualization of neurons and their synaptic connections on a small MEA with 10 neurons. Black and red lines indicate axons and dendrites respectively and green triangles indicate synaptic connections found between neurons.

${ }^{4}$ https://github.com/Vafa-Andalibi/SiMEA 


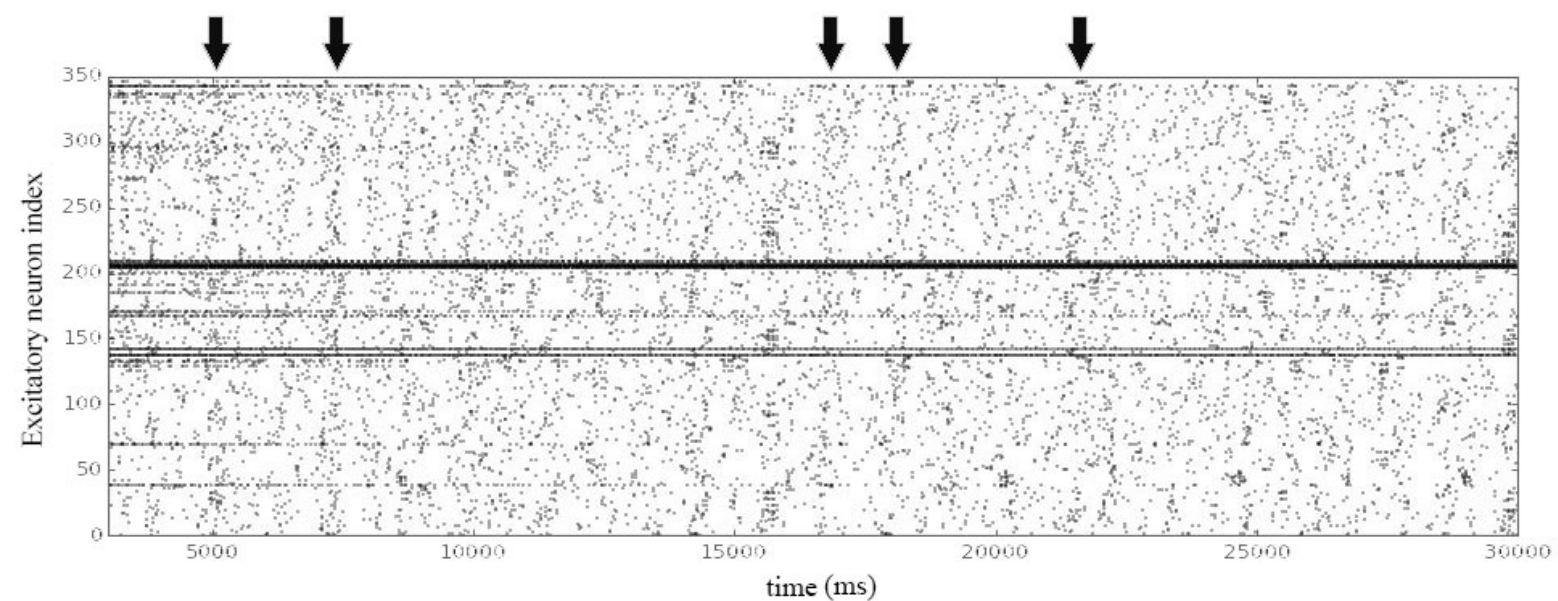

Figure 4. Output of the SiMEA in form of spikes from a simulated MEA initialized with 1000 neurons. 347 excitatory pyramidal cells, shown in the figure, and 146 inhibitory fast-spiking basket cells survived after applying the cell death. Black arrows indicate the intrinsic synchronous activity of excitatory neurons in the final output.

include the option to simulate the effect of stimulation from the MEA to the network.

\section{ACKNOWLEDGMENT}

This work is supported by the Academy of Finland under the project "Bio-integrated Software Development for Adaptive Sensor Networks", Project nupmber 278882. We would also like to show our gratitude to Dr. Simo Vanni for sharing his pearls of wisdom with us during the course of this research.

\section{REFERENCES}

[1] L. M. A. Bettencourt, G. J. Stephens, M. I. Ham, and G. W. Gross, "Functional structure of cortical neuronal networks grown in vitro," Phys. Rev. E, vol. 75, no. 2, p. 021915, 2007.

[2] Z. C. Chao, D. J. Bakkum, and S. M. Potter, "Region-specific network plasticity in simulated and living cortical networks: comparison of the center of activity trajectory (CAT) with other statistics," J. Neural Eng., vol. 4, no. 3, p. 294, 2007.

[3] M. E. Obien, K. Deligkaris, T. Bullmann, D. J. Bakkum, and U. Frey, "Revealing neuronal function through microelectrode array recordings," Front. Neurosci., vol. 8, p. 423, 2015.

[4] T. B. DeMarse, D. A. Wagenaar, A. W. Blau, and S. M. Potter, "The neurally controlled animat: Biological brains acting with simulated bodies," Auton. Robots, vol. 11, pp. 305-310, 2001.

[5] S. A. Chong, I. Benilova, H. Shaban, B. De Strooper, H. Devijver, D. Moechars, W. Eberle, C. Bartic, F. Van Leuven, and G. Callewaert, "Synaptic dysfunction in hippocampus of transgenic mouse models of Alzheimer's disease: A multi-electrode array study," Neurobiol. Dis., vol. 44, no. 3, pp. 284-291, 2011.

[6] J. Cang, R. C. Renter'lia, M. Kaneko, X. Liu, D. R. Copenhagen, and M. P. Stryker, "Development of precise maps in visual cortex requires patterned spontaneous activity in the retina," Neuron, vol. 48, no. 5, pp. 797-809, 2005.

[7] F. Christophe, V. Andalibi, T. Laukkarinen, T. Mikkonen, and K. Koskimies, "Survey and evaluation of neural computation models for bio-integrated systems," Nano Commun. Netw., vol. 6, no. 4 pp. 155-165, 2015.

[8] G. Shahaf, D. Eytan, A. Gal, E. Kermany, V. Lyakhov, C Zrenner, and S. Marom, "Order-based representation in random networks of cortical neurons," PLoS Comput. Biol., vol. 4, no. 11, 2008.

[9] Y. Li, H. Li, and Y. Wang, "Neural-based control of a mobile robot: A test model for merging biological intelligence into mechanical system," in Information Technology and Artificial Intelligence Conference (ITAIC), 2014 IEEE 7th Joint
International, 2014, pp. 186-190.

[10] S. M. Potter and T. B. DeMarse, "A new approach to neural cell culture for long-term studies.," J. Neurosci. Methods, vol. 110, no. $1-2$, pp. 17-24, 2001.

[11] J. D. Rolston, R. E. Gross, and S. M. Potter, "NeuroRighter: Closed-loop multielectrode stimulation and recording for freely moving animals and cell cultures," in Proceedings of the 31st Annual International Conference of the IEEE Engineering in Medicine and Biology Society: Engineering the Future of Biomedicine, EMBC 2009, 2009, pp. 6489-6492.

[12] A. M. Shultz, "Modeling of the networking and activity of cultured mouse neurons for simulated experiments," Master's thesis, University of Massachusetts Lowell, 2013.

[13] Y. Xu, Y. Yang, B. Han, and P. Luo, "Simulation and measurement of extracellular microelectrode array In-Vivo," in Proceedings of the World Congress on Intelligent Control and Automation (WCICA), 2010, pp. 2959-2962.

[14] S. Martinoia, P. Massobrio, M. Bove, and G. Massobrio, "Cultured Neurons Coupled to Microelectrode Arrays: Circuit Models, Simulations and Experimental Data," IEEE Trans. Biomed. Eng., vol. 51, no. 5, pp. 859-864, 2004.

[15] P. T. Thorbergsson, M. Garwicz, J. Schouenborg, and A. J. Johansson, "Computationally efficient simulation of extracellular recordings with multielectrode arrays," J. Neurosci. Methods, vol. 211, no. 1, pp. 133-144, 2012.

[16] D. Jäckel, U. Frey, F. Franke, and A. Hierlemann, "Simulator for Realistic High-Density Microelectrode Array Signals," Front. Neuroinform., no. 22.

[17] D. F. M. Goodman and R. Brette, "The brian simulator," Frontiers in Neuroscience, vol. 3. pp. 192-197, 2009.

[18] G. S. P. Miller, "The definition and rendering of terrain maps," ACM SIGGRAPH Computer Graphics, vol. 20, no. 4. pp. 39-48, 1986

[19] J. Erickson, A. Tooker, Y. C. Tai, and J. Pine, "Caged neuron MEA: A system for long-term investigation of cultured neural network connectivity," J. Neurosci. Methods, vol. 175, no. 1, pp. $1-16,2008$.

[20] E. M. Izhikevich and G. M. Edelman, "Large-scale model of mammalian thalamocortical systems.," Proc. Natl. Acad. Sci. U. S. A., vol. 105, pp. 3593-3598, 2008.

[21] G. Q. Bi and M. M. Poo, "Synaptic modifications in cultured hippocampal neurons: dependence on spike timing, synaptic strength, and postsynaptic cell type.," J. Neurosci., vol. 18, no. 24, pp. 10464-10472, 1998.

[22] H. Markram, Spike-timing dependent plasticity. Frontiers Ebooks.

[23] "Neuro-physics: Theoretical approach and modeling of neuronal network cultures." [Online]. Available: http://www.msc.univparis-diderot.fr/spip.php?rubrique374. [Accessed: 07-Mar-2016]. 